\title{
The Relationship between Dysfunctional Beliefs and Attitudes about Sleep and Sleep Structure in Patients with Insomnia: A Controlled Study
}

\author{
Jinling Yu1, Ting Xiang2, Jiyang Pan² \\ ${ }^{1}$ School of Management, Jinan University, Guangzhou, China \\ ${ }^{2}$ The First Affiliated Hospital of Jinan University, Guangzhou, China \\ Email:yjinling@163.com
}

How to cite this paper: Yu, J. L., Xiang, T., \& Pan, J. Y. (2020). The Relationship between Dysfunctional Beliefs and Attitudes about Sleep and Sleep Structure in Patients with Insomnia: A Controlled Study. Psychology, 11, 541-549.

https://doi.org/10.4236/psych.2020.113035

Received: February 28, 2020

Accepted: March 28, 2020

Published: March 31, 2020

Copyright $\odot 2020$ by author(s) and Scientific Research Publishing Inc. This work is licensed under the Creative Commons Attribution International License (CC BY 4.0).

http://creativecommons.org/licenses/by/4.0/

\begin{abstract}
Sleep is the basic survival process of human beings. However, it is clinically observed that patients with insomnia are often accompanied by cognitive disorders related to sleep. The study explores the relationship between sleep structure changes and dysfunctional beliefs and attitudes of patients with insomnia. In this study, 35 patients with insomnia disorders and 28 normal controls were evaluated as sleep attitude and belief scales and overnight-monitor polysomnography. The results show that there are significant differences in sleep parameters between the insomnia group and the control group $(p<0.05)$. The DBAS-16 score of the insomnia group is significantly lower than that of the control group, both in terms of overall comparison and gender comparison $(p<0.05)$. And there is a significant positive correlation between DBAS-16 and sleep efficiency in patients with insomnia $(r=0.34, p<0.05)$. Among them, the estimated factors for sleep consequences are significantly positively related to sleep efficiency $(r=0.34, p<0.05)$. Therefore, this study draws the following conclusions: through the comparison, it is found that patients with insomnia disorders have unreasonable sleep beliefs, and the sleep structure is different from that of the normal control group. The unreasonable beliefs of insomnia patients are related to sleep efficiency.
\end{abstract}

\section{Keywords}

Insomnia, Dysfunctional Beliefs and Attitudes about Sleep, Sleep Structure, Polysomnograms 


\section{Introduction}

With the development of socio-economic, people face increasingly higher pressures, and it is likely to cause tensions, and anxiety, and insomnia. A World Health Organization study shows that approximately $27 \%$ of people worldwide suffer from sleep disorders. Insomnia is a common clinical disease. In the recently published Diagnostic and Statistical Manual of Mental Disorders, Fifth Edition (DSM-5), the main symptom of insomnia is that in the case of adequate sleep, the main complaint is shows dissatisfactions with sleep quantity or the quality is accompanied by one or more of the following symptoms: complaints of difficulty in falling asleep; difficulty in maintaining sleep, which is characterized by frequent awakening or difficulty falling asleep after waking up; waking up early and not falling asleep again. And it may cause clinically significant pains or lead to impairment of social functions. In terms of time frequency, it means at least three nights of sleep difficulty per week, or at least three months of sleep difficulty, excluding organic sleep disorders such as sleep apnea syndrome, narcolepsy, etc., as well as insomnia caused by other mental disorders or physical diseases.

In recent decades, researches on the sleep quality of insomnia patients have been extensive. By combing the literatures on sleep quality, it is found that the factors affecting sleep quality mainly include psychological factors, environmental factors and physiological factors. In the study of influencing factors on sleep quality, it was found that cognitive factors play an important role in the occurrence and maintenance of insomnia, and cognitive factors such as irrational beliefs about sleep are considered by most researchers as the key factor (Ellis, Hampson, \& Cropley, 2007). Patients with insomnia often show prejudice to physical health, and exaggerate slight physical symptoms. Patients with insomnia often have many bad personal convictions and attitudes, pay too much attention to sleep, worry about the continuous deterioration of sleep, and desire to get a whole night's sleep (Domestic and foreign studies have shown that patients with insomnia have obvious sleep structural disorders (Edinger \& Krystal, 2003; Sun et al., 2007). Studies show that people with insomnia often have incorrect sleep beliefs and attitudes, and these dysfunctional beliefs and attitudes about sleep can affect patients' sleep quality (Carney, Edinger, Manber, Garson, \& Segal, 2007). Current researches show that sleep attitudes and beliefs are related to sleep quality. And the unreasonable sleep beliefs are inconsistent with the relationship between subjective sleep and objective sleep. But it lacks controlled studies (Zhou, Huang, $\&$ Zhou, 2017). It can be seen that there may be a close relationship between unreasonable sleep beliefs and attitudes and sleep quality. A large part of the sleep problems of insomnia may be attributed to the distorted cognitive concepts of sleep. In order to further verify the relationship between sleep structure changes and sleep attitudes and beliefs in patients with insomnia disorders, a related controlled study is performed in this article. And it aims to provide reference for psychological intervention of insomnia. 


\section{Participants and Methods}

\subsection{Participants}

Participants are patients with insomnia who underwent polysomnograms at the South China Sleep Medicine Center of the First Hospital Affiliated to Jinan University from December 2018 to October 2019. Those who sleep well are recruited. The criteria for inclusion into the insomnia group were as follows: 1) Meet the Diagnostic and Statistical Manual of Mental Disorders in the 5th edition of the US (DSM-5); 2) Age 18 - 50 years old; 3) The Pittsburgh sleep quality index score is greater than or equal to 8 ; 4) Sign the informed consent. Patients with restless legs syndrome, sleep apnea hypopnea syndrome (AHI > 5), and other sleep disorders, as well as those with a history of major physical and cerebral organic diseases and drug, alcohol or other psychoactive substance abusers were excluded. The healthy control group was evaluated before the monitoring, and they all had good sleep and physical and mental health. A total of 35 cases were included in the insomnia disorder group, 13 males and 22 females, with an average age of 32.94 \pm 8.80 years old, and 28 normal cases in the normal control group, 10 males and 18 females, with an average age of $27.82 \pm 4.86$ years. At the time of enrollment, there was no significant difference in gender between the insomnia group and the control group, but there was a significant difference in age.

\subsection{Methods}

\subsubsection{The Pittsburgh Sleep Quality Index Scale (PSQI)}

PSQI was developed by Buysse et al. (1989), a professor of the Center for Psychiatric Sleep and Biological Rhythm Research at the University of Pittsburgh Medical Center, and is used to test the sleep status of patients with insomnia in the past month. PSQI was tested by Liu et al. (1996) for patients with insomnia disorders in China, and the results showed that the reliability and validity were good, which was suitable for patients with insomnia disorders in China.

\subsubsection{The Brief Version of Dysfunctional Beliefs and Attitudes Scale (DBAS-16)}

The DBAS is used clinically to identify irrational thoughts that affect the emotions in the brain particularly before a person falls asleep. The scale was subsequently revised to a simplified version (Morin, Vallières, \& Ivers, 2007). Validation shows good reliability and validity in Chinese ( $\mathrm{Fu}, \mathrm{Ou}, \& \mathrm{Lu}, 2014)$. The questionnaire includes 16 items, which can be divided into the following four subscales: consequences caused by insomnia (referred to as consequences), sleep concerns (referred to as worry), sleep expectations (referred to as expectations) and drugs. The scoring method for each item is a 5-point score, with a total score between 16 and 80. A lower score indicates a more serious degree of unreasonable belief.

\subsubsection{Polysomnography (PSG)}

PSG is often used to diagnose sleep disorders. It monitors the data of total sleep time (TST), sleep onset latency (SOL), percentage of each sleep phase (N1\%, 
N2\%, N3\%, and REM\%), sleep efficiency (SE), and Apnea hypopnea index (AHI). Sleep time is visually scored by professional sleep technicians on electroencephalogram (EEG), electromyography (EMG), electrooculogram (EOG), electrocardiogram (ECG), nocturnal breathing and nocturnal leg movements. It not only provides a method to assess the state of sleep and wakefulness, but also can identify the abnormal physiological events that occur during sleep, so that we can associate the main complaints of insomnia with different pathological events, and provide an objective basis for the diagnosis of sleep disorders, and identification for insomnia (Kushida et al., 2005).

\subsubsection{Experimental Procedure}

All eligible participants were told to arrive at the laboratory before 20:00. The experimental environment remained free of sound and light interference, and the temperature was about 26 degrees. On the day of sleep monitoring, participants were reminded to avoid alcohol, medication, excessive caffeine or nicotine and excessive exercise affecting the behavior of EEG activity. The specific monitoring process is as follows:

1) Participants entered the sleep center on the day of the polysomnographic monitoring. Before the monitoring, the subjects were required to fill out the basic situation scale, DBQS-16, PSQI and clean their facial skin.

2) Electrode was placed by professionals using polysomnography device. Electrode placement includes EEG electrodes, eye movement electrodes, myoelectric electrodes, ECG electrodes, chest and abdomen bands, Posture detector, finger oximeter, snout detector and snore detector. After the electrodes were placed, the impedance test was performed. The impedance of all electrodes should be less than $50 \Omega$, and the corresponding electrodes should be reset when the impedance is higher than $50 \Omega$.

3) Participants started being monitored before falling asleep. The total length of night sleep monitoring was no less than 8 hours.

4) The nighttime polysomnographic monitoring chart at $30 \mathrm{~s} /$ frame was analyzed the next day. According to the staging standards prepared by Rechtschaffen and Kales $(\mathrm{R} \& \mathrm{~K})$, professionals staged the night sleep of the participants and recorded their sleep parameters.

\subsubsection{Statistical Methods}

In this study, SPSS 21.0 software was used to analyze the results. Two analyses were undertaken: 1) the scores of dysfunctional beliefs and attitudes scale, and sleep parameters of insomnia patients and normal controls, 2) related analysis of dysfunctional beliefs and attitudes and sleep structure.

\section{Results}

\subsection{Comparison of Sleep Structure between Insomnia Disorder Group and Normal Control Group}

Patients with insomnia disorders had shorter total sleep time $(t=-2.11, p<$ $0.05)$, lower sleep efficiency $(t=3.10, p<0.01)$, lower sleep III ratio $(t=-2.15, p$ 
$<0.05)$ and lower rapid eye movement sleep ratio $(t=-1.97, p<0.05)$ than those with good sleep, while sleep onset latency $(t=2.25, p<0.05)$ and wake time after sleep onset $(t=3.28, p<0.01)$ were longer, and sleep I ratio $(t=2.25$, $p<0.05)$ higher than those with good sleep. See Table 1 for details.

\subsection{Comparison of DBAS-16 Scores between Insomnia Disorder Group and Normal Control Group}

The total scores of DBAS-16 and its subscale scores in the control group were higher than those in the insomnia group, and the differences were statistically significant $(p<0.05)$. In the gender comparison, both men and women had lower scores in the insomnia group than in the control group $(p<0.01)$. This suggests more unreasonable beliefs about sleep in patients with insomnia. See Table 2 for details.

Table 1. Comparison of sleep structure between insomnia group and control group $(\bar{x} \pm s)$.

\begin{tabular}{|c|c|c|c|c|}
\hline & $\begin{array}{l}\text { Insomnia group } \\
\quad(\mathrm{n}=35)\end{array}$ & $\begin{array}{l}\text { Control group } \\
\quad(\mathrm{n}=28)\end{array}$ & $t$ & $p$ \\
\hline WB & $28.37 \pm 12.60$ & $22.44 \pm 7.25$ & 2.33 & 0.024 \\
\hline WASO & $90.17 \pm 53.39$ & $50.78 \pm 36.67$ & 3.28 & 0.002 \\
\hline SOL & $23.27 \pm 31.31$ & $10.78 \pm 9.31$ & 2.25 & 0.030 \\
\hline REMSOL & $132.32 \pm 54.66$ & $116.50 \pm 61.38$ & 1.07 & 0.288 \\
\hline SE & $76.64 \pm 14.40$ & $86.50 \pm 9.21$ & -3.10 & 0.003 \\
\hline TST & $361.01 \pm 89.26$ & $403.91 \pm 65.74$ & -2.11 & 0.039 \\
\hline N1 (\%) & $12.62 \pm 6.72$ & $9.61 \pm 3.70$ & 2.24 & 0.029 \\
\hline N2 (\%) & $50.34 \pm 8.85$ & $47.24 \pm 7.20$ & 1.33 & 0.189 \\
\hline N3 (\%) & $19.00 \pm 6.16$ & $23.27 \pm 7.20$ & -2.15 & 0.036 \\
\hline $\mathrm{R}(\%)$ & $18.45 \pm 5.74$ & $21.03 \pm 4.14$ & -1.97 & 0.053 \\
\hline
\end{tabular}

Note: $\mathrm{WB}=$ number of wake bouts, TST = total sleep time, SOL = Sleep Onset Latency, SE = Sleep Efficiency, N1 = sleep I, N2 = sleep II, N3 = sleep III, R = Rapid eye movement sleep, WASO = Wake after Sleep Onset, REMSOL = Rapid Eye Movement Sleep Onset Latency.

Table 2. Comparison of DBAS-16 between insomnia group and control group $(\bar{x} \pm s)$.

\begin{tabular}{cccccccccc}
\hline & \multicolumn{2}{c}{ Total } & \multicolumn{2}{c}{ Male } & \multicolumn{3}{c}{ Female } \\
\cline { 2 - 9 } & IG $(\mathrm{n}=35)$ & CG $(\mathrm{n}=28)$ & $t$ & IG $(\mathrm{n}=12)$ & HG $(\mathrm{n}=11)$ & $t$ & IG $(\mathrm{n}=23)$ & HG $(\mathrm{n}=17)$ & $t$ \\
\hline DBAS & $37.37 \pm 8.29$ & $49.82 \pm 10.03$ & $-5.39^{* *}$ & $36.83 \pm 9.33$ & $51.27 \pm 13.51$ & $-3.01^{* *}$ & $37.65 \pm 7.90$ & $48.88 \pm 7.32$ & $-4.58^{* *}$ \\
C & $10.60 \pm 3.31$ & $14.82 \pm 3.18$ & $-5.11^{* *}$ & $10.92 \pm 3.63$ & $14.91 \pm 4.16$ & $-2.46^{* *}$ & $10.44 \pm 3.20$ & $14.77 \pm 2.51$ & $-4.62^{* *}$ \\
W & $13.11 \pm 3.56$ & $17.89 \pm 4.17$ & $-4.90^{* *}$ & $13.17 \pm 3.54$ & $18.91 \pm 4.99$ & $-3.21^{* *}$ & $13.09 \pm 3.65$ & $17.24 \pm 3.56$ & $-3.59^{* *}$ \\
E & $4.11 \pm 1.62$ & $6.07 \pm 2.03$ & $-4.25^{* *}$ & $3.83 \pm 1.47$ & $6.55 \pm 2.38$ & $-3.25^{* *}$ & $4.26 \pm 1.71$ & $5.77 \pm 1.78$ & $-2.70^{* *}$ \\
D & $9.66 \pm 1.90$ & $11.75 \pm 1.82$ & $-4.44^{* *}$ & $9.25 \pm 2.30$ & $11.82 \pm 2.51$ & $-2.55^{* *}$ & $9.87 \pm 1.66$ & $11.71 \pm 1.26$ & $-4.58^{* *}$ \\
\hline
\end{tabular}

Note: ${ }^{*}=p<0.05,{ }^{* *}=p<0.01$, Same as below, IG = Insomnia Group, CG = Control Group, DBAS = Dysfunctional Beliefs and Attitudes Scale, C $=$ consequence, $\mathrm{W}=$ Worry, $\mathrm{E}=$ expectation, $\mathrm{D}=$ Drug. 


\subsection{Correlation Analysis between DBAS-16 Score and Sleep Structure in Patients with Insomnia}

There was a significant positive correlation between DBAS-16 and sleep efficiency in patients with insomnia $(r=0.34, p<0.05)$. Among them, the estimated factors for sleep consequences were significantly positively related to sleep efficiency $(r=0.34, p<0.05)$.Other factors were not significantly related to sleep parameters. See Table 3 for details.

\section{Discussion and Significance}

Earlier studies confirmed that cognitive disorders and physiological dysfunctions in patients with insomnia may affect sleep quality. Compared with physical disorders, cognitive disorders have a greater impact on insomnia (Lichstein \& Rosenthal, 1980). Therefore, according to the insomnia 3P model proposed by Spielman, persistent insomnia is the result of a combination of predisposing factor, precipitating factor, and perpetuating factor. Spielman et al. (1988) emphasizes that susceptible factors such as personality characteristics are the basis of insomnia, and maintenance factors such as incorrect sleep cognition are the key factors for long-term insomnia. Therefore, understanding and evaluating the impact of sleep beliefs and attitudes on sleep quality has important clinical significance for the diagnosis and treatment of insomnia disorders. This study shows that the insomnia disorder group's scores on the total score and on each subscale are significantly lower than the healthy control group, which indicates that insomnia patients are more likely to agree with unreasonable beliefs and attitudes related to sleep than healthy people, It has been confirmed that bad sleep beliefs and attitudes play an important role in the occurrence and development of insomnia.

Table 3. Correlation matrix of DBAS-16 score and sleep structure in patients with insomnia (r).

\begin{tabular}{cccccc}
\hline & DBAS-16 & Consequence & Worry & Expectation & Drug \\
\hline WB & -0.09 & 0.18 & 0.19 & -0.16 & -0.13 \\
WASO & -0.13 & -0.12 & -0.10 & -0.16 & -0.03 \\
SOL & -0.17 & -0.25 & -0.12 & -0.03 & -0.11 \\
REMSOL & 0.08 & -0.00 & 0.15 & -0.20 & 0.16 \\
SE & $0.34^{*}$ & $0.34^{*}$ & 0.26 & 0.23 & 0.23 \\
TST & 0.29 & 0.27 & 0.24 & 0.20 & 0.19 \\
N1 (\%) & -0.08 & -0.07 & -0.01 & -0.23 & -0.25 \\
N2 (\%) & 0.11 & 0.10 & 0.12 & -0.02 & -0.07 \\
N3 (\%) & -0.05 & -0.15 & -0.07 & 0.08 & 0.13 \\
R (\%) & 0.03 & 0.03 & -0.05 & 0.18 & -0.01 \\
\hline
\end{tabular}

Note: WB = number of wake bouts, TST = total sleep time, SOL = Sleep Onset Latency, SE = Sleep efficiency, N1 = sleep I, N2 = sleep II, N3 = sleep III, R = Rapid eye movement sleep, WASO = Wake after Sleep Onset, REMSOL = Rapid Eye Movement Sleep Onset Latency. 
Some studies at home and abroad on polysomnography in patients with insomnia disorders have found that patients with insomnia have prolonged sleep latency, reduced total sleep time, decreased sleep efficiency, increased wakefulness after falling asleep, increased light sleep, and deep sleep (Pigeon \& Perlis, 2006; Pan, Yao, \& Li, 2013). The characteristics of polysomnography in patients with insomnia disorders obtained in this study are basically consistent with the results of previous studies. Patients had reduced total sleep time, decreased sleep efficiency, prolonged sleep latency, increased waking time and frequency after falling asleep, increased proportion of sleep I, and decreased proportion of sleep III time. The number of wake bouts in the insomnia disorder group was significantly higher than that in the normal control group, indicating that patients with insomnia have poor sleep stability. And there may be defects in sleep microstructure and sleep homeostasis, easily causing wakefulness.

The results of this study show that false beliefs about sleep lead to reduced sleep efficiency. Studies have shown that changing personal beliefs and attitudes helps improve sleep efficiency (Eidelman et al., 2015). Numerous research data show that cognitive behavioral treatment of insomnia can significantly improve sleep quality in patients with insomnia (Dolan, Taylor, Bramoweth, \& Rosenthal, 2010; Cervena et al., 2004). In particular, health education can change the misunderstanding of patients with insomnia, and significantly improve the quality of sleep and quality of life of patients with insomnia (Chen, 2008).

Sleep problems are already one of the most important issues affecting quality of life for most people. There are many researches on sleep cognitive behaviors in foreign countries, and the theoretical models of sleep are relatively mature. But Chinese researches in this filed is just in its infancy. This study explores the cognitive factors that affect sleep quality to enrich Chinese theories on sleep cognition and behaviors, and provide a theoretical support and an empirical evidence for researches on improving sleep quality through cognitive and behavioral therapy. Furthermore, this study explores the specific status of dysfunctional beliefs and attitudes and objective sleep quality, and the ways how the dysfunctional beliefs and attitudes affect sleep quality. It helps insomniac patients to understand the cognitive roots of their sleep problems and facilitates early identification, objective diagnosis, and early intervention of poor sleep habits in patients with insomnia disorders. In this way, these adverse factors may be improved and patients' sleep quality may be increased.

\section{Deficiencies and Prospects}

The results of this study effectively extend researches on sleep quality of patients with insomnia disorders, provide important theoretical guidance for the psychological treatment of insomnia, and provide a basis for cognitive behavioral treatment of insomnia. However, the number of samples in this study is small and tends to be younger. The insomnia group and the normal control group do not match in age. Although the sleep problem is getting younger and younger, 
for improving the universality of the conclusion, subsequent researches should still involve all ages, and in control studies, gender and age should be matched. Secondly, there are various factors affecting sleep quality, among which the mechanism of action is complex. This article only explores the relationship between unreasonable sleep beliefs and sleep structures from a cognitive perspective, and proposes an explanation of the factors affecting sleep quality. In the end, this study was conducted theoretically without interventions. In the future, researchers can intervene in patients' poor cognition and observe the differences before and after the intervention. Since unreasonable sleep belief is an important factor affecting insomnia, education can be used to improve patients' awareness of sleep problems, alleviate insomnia symptoms, and promote the sleep quality of patients with insomnia disorders.

\section{Conflicts of Interest}

The authors declare no conflicts of interest regarding the publication of this paper.

\section{References}

Buysse, D. J., Iii, C. F. R., Monk, T. H., Berman, S. R., \& Kupfer, D. J. (1989). The Pittsburgh Sleep Quality Index: A New Instrument for Psychiatric Practice and Research. Psychiatry Research, 28, 193-213. https://doi.org/10.1016/0165-1781(89)90047-4

Carney, C. E., Edinger, J. D., Manber, R., Garson, C., \& Segal, Z. V. (2007). Beliefs about Sleep in Disorders Characterized by Sleep and Mood Disturbance. Journal of Psychosomatic Research, 62, 179-188. https://doi.org/10.1016/j.jpsychores.2006.08.006

Cervena, K., Dauvilliers, Y., Espa, F., Touchon, J., Matousek, M., Billiard, M. et al. (2004). Effect of Cognitive Behavioural Therapy for Insomnia on Sleep Architecture and Sleep EEG Power Spectra in Psychophysiological Insomnia. Journal of Sleep Research, 13, 385-393. https://doi.org/10.1111/j.1365-2869.2004.00431.x

Chen, X. M. (2008). Application of Sleep Belief and Attitude Scale in Health Education for Patients with Insomnia. Chinese Journal of Mental Health, 22, 833-836. (In Chinese)

Dolan, D. C., Taylor, D. J., Bramoweth, A. D., \& Rosenthal, L. D. (2010). Cognitive-Behavioral Therapy of Insomnia: A Clinical Case Series Study of Patients with Co-Morbid Disorders and Using Hypnotic Medications. Behaviour Research \& Therapy, 48, 321-327. https://doi.org/10.1016/j.brat.2009.12.004

Edinger, J. D., \& Krystal, A. D. (2003). Subtyping Primary Insomnia: Is Sleep State Misperception a Distinct Clinical Entity? Sleep Medicine Reviews, 7, 203-214. https://doi.org/10.1053/smrv.2002.0253

Eidelman, P., Talbot, L., Ivers, H., Bélanger, L., \& Harvey, A. G. (2015). Change in Dysfunctional Beliefs about Sleep in Behavior Therapy, Cognitive Therapy, and Cognitive-Behavioral Therapy for Insomnia. Behavior Therapy, 47, 102-115. https://doi.org/10.1016/j.beth.2015.10.002

Ellis, J., Hampson, S. E., \& Cropley, M. (2007). The Role of Dysfunctional Beliefs and Attitudes in Late-Life Insomnia. Journal of Psychosomatic Research, 62, 81-84. https://doi.org/10.1016/j.jpsychores.2006.06.007

Fu, S. X., Ou, H. X., \& Lu, S. H. (2014). Reliability and Validity of the Short Sleep Belief and Attitude Scale. Chinese Journal of Behavioral Medicine and Brain Science, 23, 369-371. (In Chinese) 
Kushida, C. A., Littner, M. R., Morgenthaler, T., Alessi, C. A., Bailey, D., Coleman, J. et al. (2005). Practice Parameters for the Indications for Polysomnography and Related Procedures: An Update for 2005. Sleep, 28, 499-523. https://doi.org/10.1093/sleep/28.4.499

Lichstein, K. L., \& Rosenthal, T. L. (1980). Insomniacs' Perceptions of Cognitive versus Somatic Determinants of Sleep Disturbance. Journal of Abnormal Psychology, 89, 105-107. https://doi.org/10.1037/0021-843X.89.1.105

Liu, X. C., Tang, M. Q., Hu, L., Wang, A. Z., Wu, H. X., Zhao, G. F., Gao, C. N., \& Li, W. S. (1996). Reliability and Validity of Sleep Quality Index of Pitzburg. Chinese Journal of Psychiatry, 29, 103-107. (In Chinese)

Morin, C. M., Vallières, A., \& Ivers, H. (2007). Dysfunctional Beliefs and Attitudes about Sleep (DBAS): Validation of a Brief Version (DBAS-16). Sleep, 30, 1547-1554. https://doi.org/10.1093/sleep/30.11.1547

Pan, S. K., Yao, P. F., \& Li, H. F. (2013). Research and Related Analysis of Polysomnographic EEG in Primary Insomnia. Journal of Psychiatry, 26, 391-393. (In Chinese)

Pigeon, W. R., \& Perlis, M. L. (2006). Sleep Homeostasis in Primary Insomnia. Sleep Medicine Reviews, 10, 247-254. https://doi.org/10.1016/j.smrv.2005.09.002

Spielman, A. J., Caruso, L. S., \& Glovinsky, P. B. (1988). A Behavioral Perspective on Insomnia. Psychiatric Clinics of North America, 10, 541-553. https://doi.org/10.1016/S0193-953X(18)30532-X

Sun, Y., Yang, Z. J., Gu, Y., Shen, Y., Fan, D. S., \& Lv, J. Q. (2007). Subjective Assessment of Sleep Quality in Patients with Insomnia and Comparative Analysis of Polysomnographic Parameters. Chinese Journal of Neurology, 40, 498-500. (In Chinese)

Zhou, X. Y., Huang, G. P., \& Zhou, Y. F. (2017). Relationship between Beliefs and Attitudes about Sleep and Objective/Subjective Sleep. Mental Health of Sichuan, 30, 528-531. (In Chinese) 